\title{
1-GHz all-optical flip-flop operation of conventional cylindrical-shaped single-mode VCSELs under low-power optical injection.
}

Lee, S.H.; Jung, H.W.; Kim, K.H.; Lee, M.H.; Yo, B.S.; Roh, J.; Shore, K.A.

\section{IEEE Photonics Technology Letters}

\author{
DOI: \\ 10.1109/LPT.2010.2078803
}

Published: 01/12/2010

Peer reviewed version

Cyswllt i'r cyhoeddiad / Link to publication

Dyfyniad o'r fersiwn a gyhoeddwyd / Citation for published version (APA):

Lee, S. H., Jung, H. W., Kim, K. H., Lee, M. H., Yo, B. S., Roh, J., \& Shore, K. A. (2010). 1-GHz all-optical flip-flop operation of conventional cylindrical-shaped single-mode VCSELs under lowpower optical injection. IEEE Photonics Technology Letters, 22(23), 1759-1761.

https://doi.org/10.1109/LPT.2010.2078803

\section{Hawliau Cyffredinol / General rights}

Copyright and moral rights for the publications made accessible in the public portal are retained by the authors and/or other copyright owners and it is a condition of accessing publications that users recognise and abide by the legal requirements associated with these rights. study or research.

- Users may download and print one copy of any publication from the public portal for the purpose of private

- You may not further distribute the material or use it for any profit-making activity or commercial gain

- You may freely distribute the URL identifying the publication in the public portal ?

Take down policy

(c) 2010 IEEE. Personal use of this material is permitted. Permission from IEEE must be obtained for all other users, including reprinting/ republishing this material for advertising or promotional purposes, creating new collective works for resale or redistribution to servers or lists, or reuse of any copyrighted components of this work in other works

Take down policy

If you believe that this document breaches copyright please contact us providing details, and we will remove access to the work immediately and investigate your claim. 


\title{
1-GHz All-Optical Flip-Flop Operation of Conventional Cylindrical-Shaped Single- Mode VCSELs Under Low Power Optical Injection
}

Seoung Hun Lee, Hae won Jung, Kyong Hon Kim, Member, IEEE, Min Hee Lee, Byeung-Soo Yoo,

Jay Roh, and K. Alan Shore, Senior Member, IEEE

\begin{abstract}
All-optical set-reset flip-flop operations of conventional circular cylindrical-shaped 1.55- $\mu \mathrm{m}$ wavelength single-mode vertical-cavity surface-emitting lasers (SM VCSELs) have been demonstrated at a switching frequency of $1 \mathrm{GHz}$ based on polarization bistability observed via injection current variation. The energy of set and reset pulses was lower than $4.5 \mathrm{fJ}$ at a bias current of the VCSEL of $4 \mathrm{~mA}$. Polarization bistable VCSELs will be useful for highspeed optical signal processing applications.
\end{abstract}

Index Terms - Flip-flops, optical bistability, optical injection, polarization switching, vertical-cavity surfaceemitting lasers (VCSELs).

\section{INTRODUCTION}

$\mathrm{A}$ LL-OPTICAL flip-flops (AOFFs) using optical bistable devices can be used for many applications in future high-speed optical networks and optical computing, such as optical switches, optical memories and optical clock generation [1]-[3]. InP-based semiconductor devices such as semiconductor optical amplifiers, distributed feedback laser diodes, Fabry-Perot LDs and micro-disk lasers have been used for demonstration of the AOFF by various research groups[4]-[7]. Low power consumption, high-speed operation and ability for photonic integration of such devices are very important for future practical applications. Recently small and low power flip-flop operation of a hybrid InP integrated micro-disk laser on a silicon-on-insulator (SOI) wafer was reported with low electrical bias current and injected switching pulse energy of $3.5 \mathrm{~mA}$ and 1.8 fJ, respectively [7]. However, the hybrid integrated device has a significant coupling loss between the InP device, SOI waveguide, and coupled optical fibers.

VCSELs have been recognized as useful optical devices because of their potential for integration in 2dimensional arrays, low power consumption, low coupling loss to optical fibers and low cost compared to other devices. Several research reports on the optical bistability of the VCSELs and applications to the AOFF operation have been made previously. However, previous reports are mostly related to observations of optical bistability and AOFF operation with 850-nm or 980-nm wavelength VCSELs [8], [9]. In the optical telecom wavelength region of $1550 \mathrm{~nm}$, AOFF operation based on polarization bistability of a specially designed VCSEL with square-shaped mesa structure was demonstrated, but the optical bistability was observed at a relatively high-operating current of above $14.1 \mathrm{~mA}$ [10]. Optical injection induced polarization bistability in commercially available single-mode VCSELs has also been reported, but the injection power required for a hysteresis curve between the two orthogonal polarization states was several hundred microwatt of a continuouswave mode laser beam [11],[12]. Recently we have observed operating current-induced polarization bistability of conventional cylindrical-shaped single-mode VCSELs at $1.55 \mu \mathrm{m}$ wavelength, and have also reported a potential for all-optical flip-flop operation with the polarization bistability of the VCSEL [13].

In this letter, we report 1-GHz all-optical flip-flop operation using the polarization bistability of conventional circular cylindrical-shaped 1550-nm wavelength single-mode VCSELs with a low switching energy of $4.5 \mathrm{fJ}$.

\section{EXPERIMENTS AND RESULTS}

In this experiment we used commercially available single longitudinal-mode $1.55-\mu \mathrm{m}$ wavelength VCSELs with circular cylindrical shaped cavity (Raycan). VCSELs with ideal circular symmetry output surface structure 
can have fundamental transverse mode lasing output in one of two orthogonal linear polarization states, whose directions are aligned with the $\langle 110\rangle$ and $\langle 1 \overline{1} 0\rangle$ crystal directions of the InAlGaAs/InP compound semiconductor materials. However, due to device asymmetries, birefringence and anisotropy the SM VCSELs deliver a laser output at a well-defined polarization direction for injection currents above threshold current, and show polarization switching and bistability as the injection current varies. The polarization switching and bistability properties of the VCSELs varied from chip to chip even within one wafer, which might depend on amount of anisotropic stresses built up inside each of the VCSEL chips during the fabrication process [13].

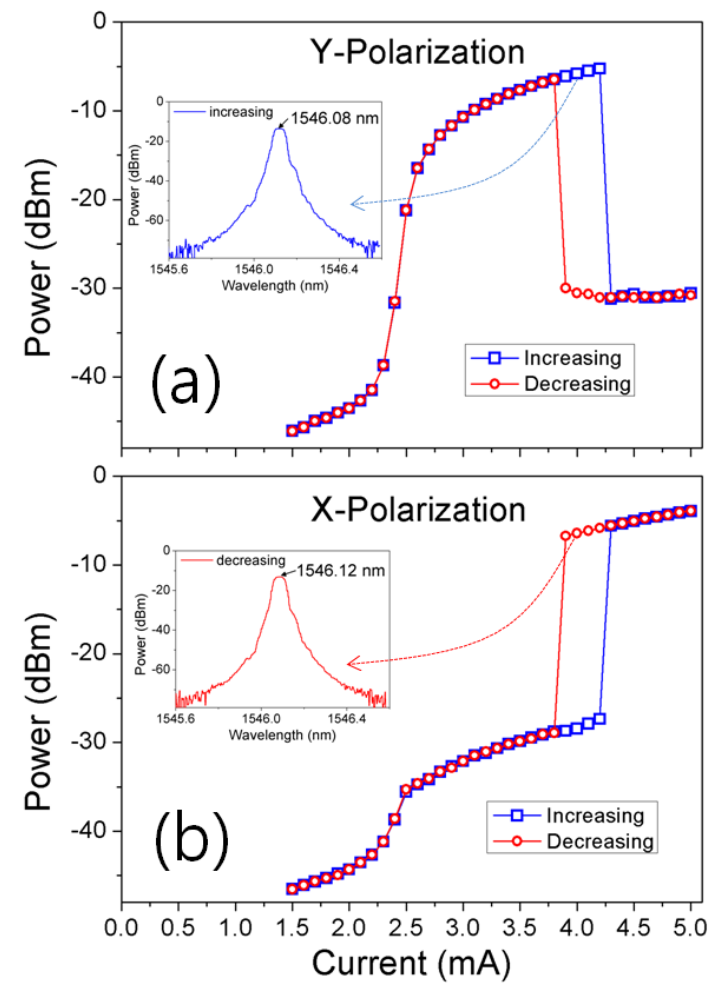

Fig. 1. Measured L-I curves of the bistable VCSEL in (a) Y-and (b) Xpolarization directions. Inset: optical spectra of the VCSEL's output at $4 \mathrm{~mA}$ with (a) increasing bias current and (b) decreasing bias current.

Fig. 1 shows the polarization switching and bistability conditions in the $L-I$ curve of a SM VCSEL versus increasing and decreasing bias current - obtained using the measurement setup shown in Fig. 2. The VCSEL had a threshold current of $2.4 \mathrm{~mA}$ at $17.4 \pm 0.002{ }^{\circ} \mathrm{C}$. The VCSEL's temperature was controlled with a thermoelectric cooler packaged in its TO-can package. Two different polarization switching currents were observed at a $0.5 \mathrm{~mA}$ spacing as shown in Fig.1. The first lasing mode appeared at an initial polarization state, called the Ypolarization mode. As the bias current was increased above $4.3 \mathrm{~mA}$, the output switched abruptly to the orthogonal polarization state, called the X-polarization mode. On the other hand, as the bias current was decreased from a high current, the X-polarization mode output switched back to the Y-polarization mode output at $3.8 \mathrm{~mA}$. The insets of Figs. 1 (a) and (b) represent optical spectra measured with an optical spectrum analyzer (OSA) at a bias current of $4.0 \mathrm{~mA}$ as the current was increased and decreased, respectively. The corresponding VCSEL output powers were $-9.6 \mathrm{dBm}$ in the Y-polarization and $-10.0 \mathrm{dBm}$ in the Xpolarization for increasing and decreasing current respectively. The wavelength separation of the spectral peaks between the two orthogonal polarization states was about $0.04 \mathrm{~nm}$. 


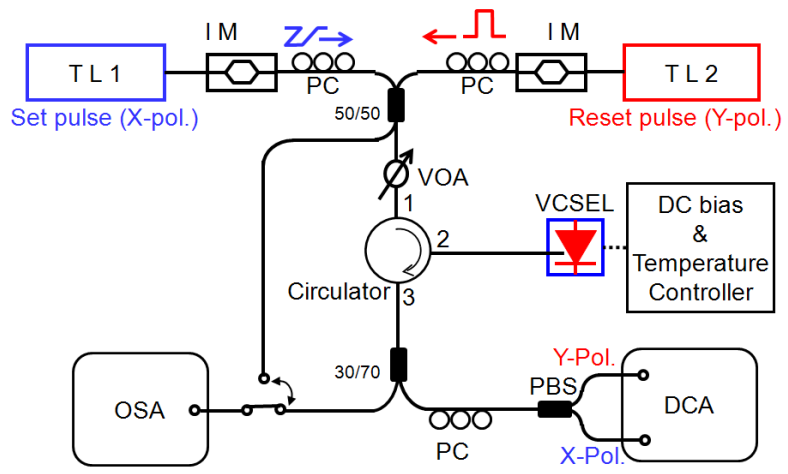

Fig. 2. Experimental setup for all-optical flip-flop operation with injection of set and reset pulses.

Polarization bistability based AOFF operations were performed with the experimental setup shown in Fig. 2 . Modulated signal pulses from two tunable lasers (TLs) with Mach-Zehnder intensity modulators (IMs) were used for the set and reset optical pulses. The intensity modulators (IMs) were driven with a $3.2 \mathrm{GHz}$ pulse pattern generator. The set and reset pulses were passed through a 50/50 directional coupler, a variable optical attenuator (VOA) and an optical circulator, and then injected into the VCSEL. The output from the VCSEL passed through the same optical circulator, a 30/70 directional coupler, another polarization controller, and a fiber-type polarization beam splitter (PBS). Two separated orthogonal polarization output from the PBS were measured with a digital communication analyzer (DCA) with two $20 \mathrm{GHz}$ photoreceiver modules.

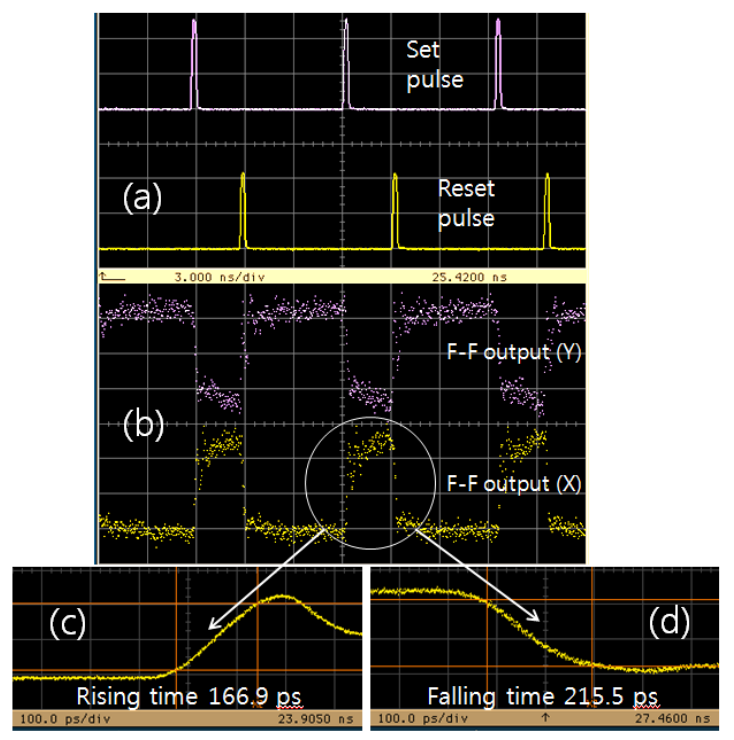

Fig. 3. Waveforms of the (a) set /reset pulses and (b) flip-flop output at a 346 $\mathrm{MHz}$ switching frequency. (c) and (d) show the rise and fall times of the switch-on and switch-off transients, respectively.

We first performed a low repetition rate AOFF operation to test the switching time. The VCSEL was operated at a driving current of $4.0 \mathrm{~mA}$ which was within the bistable region. Initially the VCSEL's output was adjusted to deliver a Y-polarized output at $1546.08 \mathrm{~nm}$. The wavelengths of TL 1 (set) and 2 (reset) were adjusted to about 1546.12 and $1546.08 \mathrm{~nm}$, respectively, which corresponded to the peak wavelengths of the Xand Y-polarization outputs as shown in Fig. 1. Fig. 3 (a) shows the measured pulse patterns of injected set and reset pulses, each of which had a pulse width of $280 \mathrm{ps}$ and a rising time of $112 \mathrm{ps}$. When a set pulse stream in the X-polarization direction was injected into the VCSEL, the output was switched from the Y-polarization state to the X-polarization state at a wavelength of $1546.12 \mathrm{~nm}$, and then was kept in the switched X-polarization state. For injection of the reset pulses in the Y-polarization direction the X-polarization output was returned 
back to the Y-polarization as shown in Fig. 3(b). Figs. 3 (c) and (d) show the switch-on and switch-off times of the AOFF signals, each of which are $166.9 \mathrm{ps}$ and $215.5 \mathrm{ps}$, respectively. These temporal response times might be reduced further by using injection pulses of short pulse width as shown in Ref. 5. Based on the measured switch-on and switch-off times the VCSEL can be used for AOFF operation at frequencies greater than $2 \mathrm{GHz}$.

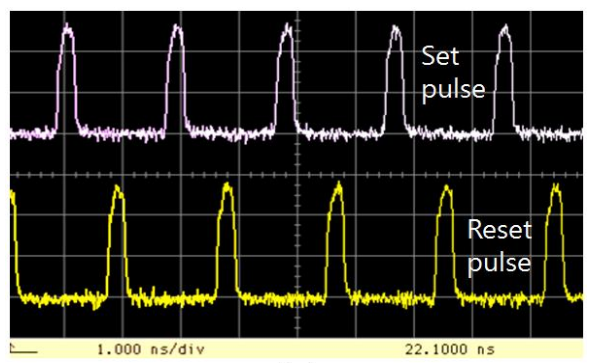

(a)

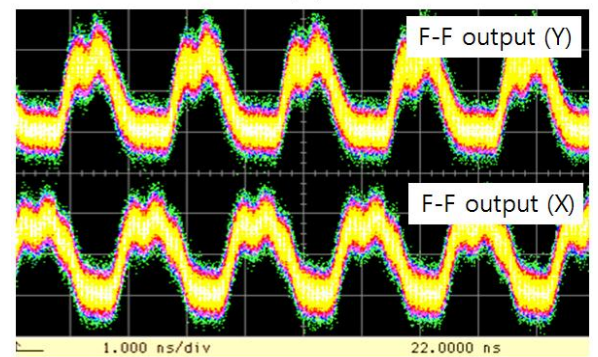

(b)

Fig. 4. All-optical flip-flop operation for $1 \mathrm{GHz}$ switching frequency: (a) the set and reset signals injected into the VCSEL and (b) oscilloscope traces of the flip-flop outputs accumulated for an acquisition time of $20 \mathrm{sec}$.

Fig. 4 shows the measured waveforms of the AOFF operation of the SM VCSEL at a switching frequency of about $1 \mathrm{GHz}$. The injected pulse energies of the set and reset pulses into the VCSEL were only $4.5 \mathrm{fJ}$ and $3.5 \mathrm{fJ}$, respectively. These values of the injection pulse energies for the flip-flop operation are less than those reported in Ref. 10 where a VCSEL of square-shaped mesa structure was used instead of the conventional circular cylindrical-shaped VCSELs. The operation speed can be further enhanced if very short injection pulses are used.

\section{CONCLUSION}

We have demonstrated $1 \mathrm{GHz}$ AOFF operation of a conventional circular cylindrical-shaped 1.55- $\mu \mathrm{m}$ wavelength SM VCSEL under low power optical injection based on its polarization bistability. The pulse energy of set/reset signals was smaller than $4.5 \mathrm{fJ}$, and the bias current of the VCSEL was $4 \mathrm{~mA}$. Further improvement of switching speed of the flop-flop operation can be achieved with high speed pulse generators. AOFF operation based on polarization bistable VCSELs will be useful for cost-effective applications in high-speed signal processing in future optical switching, optical router, optical memory, and optical computing.

This work was supported in part by the Basic Science Research Programs through the National Research Foundation of Korea (NRF) funded by the Korean Ministry of Education, Science and Technology under Grants 2009-0073617, 2009-0084514 and $2010-0001474$.

Seoung Hun Lee, Hae Won Jung, Kyong Hon Kim and Min Hee Lee are now with the Department of Physics, Inha University, Incheon, 402-751, Republic of Korea (phone: 82-32-876-7664; fax: 82-32-872-7562; e-mail: kyongh@ inha.ac.kr).

Byeung-Su Yoo and Jay Roh are with Raycan Co., Ltd., 138 Gajeong-dong, Daejeon, 305-350, Republic of Korea

K. A. Shore is with Bangor University, School of Electronic Engineering, Bangor, LL57 1 UT, Wales, UK

\section{REFERENCES}

[1] K. Huybrechts, T. Tanemura, Y. Nakano, R. Baets, and G. Morthier, "40-Gb/s all-optical packet switching with a distributed-feedback laser as all-optical flip-flop,” IEEE Photon. Technol. Lett., vol. 21, no. 11, pp. 703-705, Jun. 2009.

[2] M. T. Hill, H. J. S. Dorren, T. De Vries, X. J. M. Leijtens, J. H. Den Besten, B. Smalbrugge, Y. -. Oel, H. Binsma, G. -. Khoe, and M. K. Smit, "A fast low-power optical memory based on coupled micro-ring lasers," Nature, vol. 432, no. 11, pp. 206-209, Nov. 2004.

[3] A. M. Kaplan, G. P. Agrawal, and D. N. Maywar, "Optical square-wave clok generation based on an all-optical flip-flop," IEEE Photon. Technol. Lett., vol. 22, no. 7, pp. 489-491, Apr. 2010.

[4] D. N. Maywar, G. P. Agrawal, and Y. Nakano, "All-ooptical hysteresis control by means of cross-phase modulation in semiconductor optical amplifiers,” J. Opt. Soc. Am. B, vol. 18, no. 7, pp. 1003-1013, Jul. 2001. 
[5] K. Huybrechts, G. Morthier, and R. Baets, "Fast all-optical flip-flop based on a single distributed feedback laser diode," Opt. Express, vol. 16, no. 15, pp. 11405-11410, Jul. 2008.

[6] Y. D. Jeong, J. S. Cho, Y. H. Won, H. J. Lee, and H. Yoo, “All-optical flip-flop based on the bistability of injection locked Fabry-perot laser diode," Opt. Express, vol. 14, no. 9, pp. 4058-4063, May 2006.

[7] L. Liu, R. Kumar, K. Huybrechts, T. Spuesens, G. Roelkens, E. -. Geluk, T. De Vries, P. Regreny, D. Van Thourhout, R. Baets, and G. Morthier, "An ultra-small, low-power, all-optical flip-flop memory on a silicon chip," Nature Photonics, vol. 4, pp. 182-187, Mar. 2010.

[8] Y. Hong, R. Ju, P. S. Spencer, and K. A. Shore, "Investigation of polarization bistability in vertical-cavity surface-emittting lasers subjected to optical feedback," IEEE J. Quant. Electron., vol. 41, no. 5, pp. 619-624, May. 2005.

[9] T. Mori, Y. Yamayoshi, and H. Kawaguchi, "Low-switching-energy and high-repetition-frequency all-optical flip-flop operations of a polarization bistable vertical-cavity surface-emitting laser," Appl. Phys. Lett., vol. 88, 101102, Mar. 2006.

[10] T. Katayama, T. Ooi, and H. Kawaguchi, "Experimental demonstration of multi-bit optical buffer memory using 1.55- $\mu$ m polarization bistable vertical-cavity surface-emitting lasers," IEEE J. Quant. Electron., vol. 45. no. 11, pp. 1495-1504, Nov. 2009.

[11] A. Hurtado, A. Quirce, A. Valle, L. Pesquera and M. J. Adams, "Power and wavelength polarization bistability with very wide hysteresis cycles in a $1550 \mathrm{~nm}$-VCSEL subject to orthogonal optical injection,” Opt. Express, vol. 17. no. 26, pp. 23637-23642, Dec. 2009.

[12] A. Quirce, A. Valle, and L. Pesquera, "Very wide hysteresis cycles in 1550-nm VCSELs subject to orthogonal optical injection," IEEE Photon. Technol. Lett., vol 21 no. 17, pp 1193-1195, Sep. 2009.

[13] S. H. Lee, H. W. Jung, K. H. Kim and M. H. Lee, "All-Optical Flip-Flop Operation Based on Polarization Bistability of ConventionalType 1.55- $\mu \mathrm{m}$ Wavelength Single-Mode VCSELs," submitted to J. of the Opt. Soc. of Korea [see OSA InfoBase later] (2010). 